\title{
FLUOROSCOPIC ANALYSIS OF KINEMATICS AFTER POSTERIOR-CRUCIATE-RETAINING KNEE ARTHROPLASTY
}

\author{
JAMES B. STIEHL, RICHARD D. KOMISTEK, DOUGLAS A. DENNIS, ROBERT D. PAXSON, \\ WILLIAM A. HOFF
}

From the Medical College of Wisconsin, Milwaukee, USA

We used fluoroscopy to study the kinematics of the knee in 47 patients with total knee arthroplasty (TKA) and four control subjects with normal knees while performing a single-leg deep-knee bend. The videos were analysed using still photographs taken at $5^{\circ}$ increments of flexion. Femorotibial contact points, patellar ligament rotation, and patellar rotation were calculated from each image. Maximum weight-bearing flexion was determined for each knee.

Compared with the control group, posteriorcruciate-retaining TKA did not reproduce normal knee kinematics in any case, but showed a starting point posterior to the tibial midline which translated anteriorly with flexion. The curves from successive knee bends could not be consistently reproduced. Under weight-bearing conditions, the maximum flexion for any PCR TKA was $98^{\circ}$ and several patients could not flex beyond $70^{\circ}$.

J Bone Joint Surg [Br] 1995;77-B:884-9.

Received 5 April 1995; Accepted 30 May 1995

J. B. Stiehl, MD, Clinical Associate Professor

Department of Orthopaedic Surgery, Medical College of Wisconsin, Milwaukee, Wisconsin 53211, USA.

R. D. Komistek, PhD, Assistant Professor

Rose Musculoskeletal Research Laboratory, Rose Medical Center, 4545 East Ninth Avenue, Denver, Colorado 80220, USA.

R. D. Paxson, MS

Pheonix Medical Technology Inc, 3477 Jenny Lane, Bartlett, Tennessee 38135, USA.

W. A. Hoff, PhD, Assistant Professor

D. A. Dennis, MD, Professor

Colorado School of Mines, Division of Engineering, Golden, Colorado 80401, USA.

Correspondence should be sent to Dr J. B. Stiehl at 2015 East Newport Avenue 703, Milwaukee, Wisconsin 53211, USA.

O1995 British Editorial Society of Bone and Joint Surgery 0301-620X/95/61094\$2.00
Attempts have been made in the past to determine the normal kinematics of the knee (Müller 1983; Wongchaisuwat, Hemami and Buchner 1984; Nisell, Nemeth and Ohlsen 1986; Yamaguchi and Zajac 1989; Hsu et al 1990; O'Connor et al 1990). Video fluoroscopy and MRI are now used to define knee geometry and kinematics in vivo (Stein and Granik 1979; Nittsu, Akisada and Miyakawa 1990; Banks and Hodge 1993). In total knee arthroplasty (TKA) this knowledge is important in the understanding of the weight-bearing forces and shear stresses applied to bearing surfaces. It is likely that abnormal kinematics is detrimental to the performance of a TKA. Excessive wear has been seen in polyethylene inserts from TKA retrievals (Grood, Shastri and Hopson 1982; Rose and Cimino 1982; Landy and Walker 1988; Collier et al 1990; Swany and Scott 1993). One hypothesis is that the structure of existing polyethylene material is inadequate and that much of the problem may be material-related.

From a mechanical point of view, low area line contact, typical of many posterior-cruciate-retaining (PCR) prosthetic designs, may cause higher localised stresses in the bearing surface leading to higher rates of wear (Collier et al 1991; Kilgus et al 1991). These stresses are a function of the load applied, the area of contact and, in this situation, the velocity of shear applied to the polyethylene surface (Brown and Shaw 1984). New designs have sought to make contact surfaces more congruent with the aim of improving the area of contact (Goodfellow and O'Connor 1986). Translation of the implant, however, can lead to a ploughing action which, when exaggerated, creates polyethylene delamination from subsurface failure of the material (Bartel, Bicknell and Wright 1986; Blunn et al 1991).

Posterior-cruciate retention in TKA has been advocated as a means of preserving anatomical femoral roll-back and potentially increasing the subject's range of motion. Using anatomical and theoretical models, several authors have agreed that roll-back is preserved after anterior-cruciate excision in PCR TKA (Andriacchi, Stanwyck and Galante 1986; El Nahass, Madson and Walker 1990; Mahoney et al 1990; Schlepckow 1992). Other authors believe that the range of movement is increased in TKA when the PCL is sacrificed and a posterior-stabilised implant is used to maintain femoral roll-back (Insall, Lachiewicz and Burstein 1982; Stern and Insall 1992). Two factors which influence the kinematics of the knee are the shape of the articular 


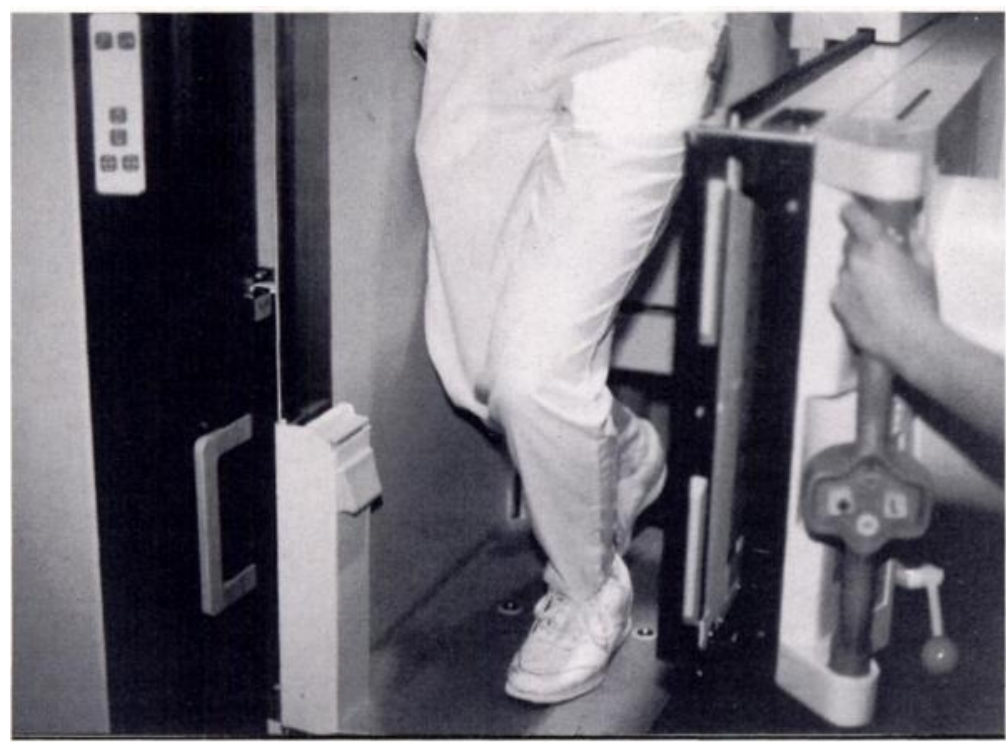

Fig. 1

Single-stance deep-knee bend during lateral-medial fluoroscopy.

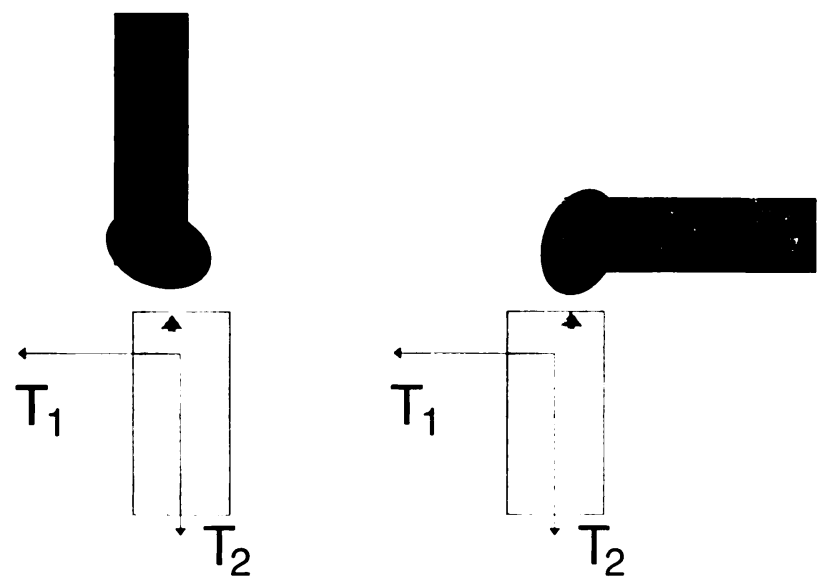

Fig. 2

The femorotibial contact position is measured with reference to the midsagittal plane of the tibia.

surfaces and the ligaments or artificial constraints. In the normal knee, a rolling motion predominates for the first $20^{\circ}$ of flexion, which produces a posterior translation of the femoral contact position on the tibia. After $30^{\circ}$ of flexion, sliding becomes predominant but the net motion remains in the posterior direction (Müller 1983).

Clinical studies have shown that PCR TKA will provide excellent long-term results and a satisfactory postoperative range of movement (Wright et al 1990; Goldberg 1991; Maloney and Schurman 1992). With these impressions in mind, we sought to study the various kinematic patterns derived under dynamic, in vivo conditions, such as femorotibial contact, weight-bearing range of movement and patellar ligament and patellar rotations.

\section{PATIENTS AND METHODS}

We performed dynamic fluoroscopy kinematically under weight-bearing conditions in 47 patients with PCR TKA and four control subjects with normal knees. The control group was filmed using a Siemens Polydoros 80S fluoroscopy machine as they performed a single-stance deep-knee bend to maximum flexion (Figs 1 and 2). The fluoroscopy machine produced images at a rate of $30 \mathrm{~Hz}$.

The patients in the TKA group were chosen on the basis of a satisfactory clinical result using the Knee Society scoring system. Pain was mild or absent in all (average 47.7 ) and the functional score averaged 90.7. Their average age was 69 years and 12 had had bilateral TKAs. Five different prosthetic designs were studied including the Porous Coated Anatomic (8) (Howmedica), Ortholoc (11) (Wright Medical Technology), Genesis (9) (Richards), Anatomic Modular Knee (10) (Depuy), and Miller-Galante 2 (9) (Zimmer).

In each patient, fluoroscopy in the sagittal plane from both the lateral and medial views was performed during three successive deep-knee bends. Each knee bore full body-weight and flexion was taken to mechanical block or pain. The video was then analysed using still photographs taken at $5^{\circ}$ increments. Data for the position of the femur 


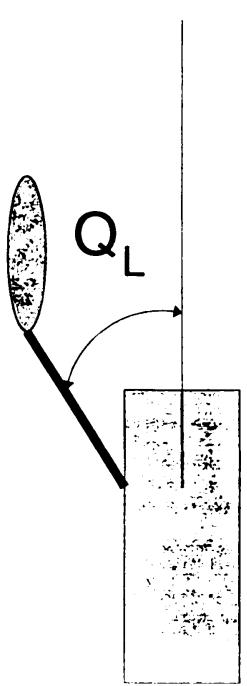

Fig. 3

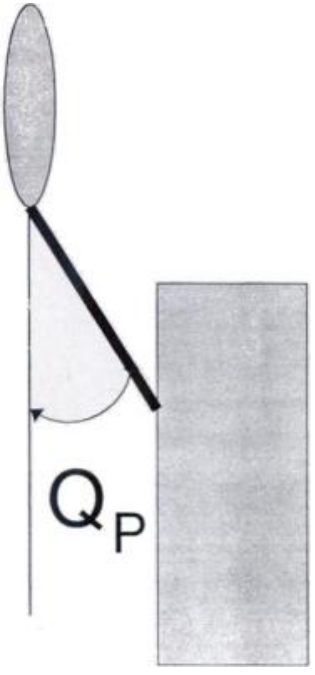

Fig. 4
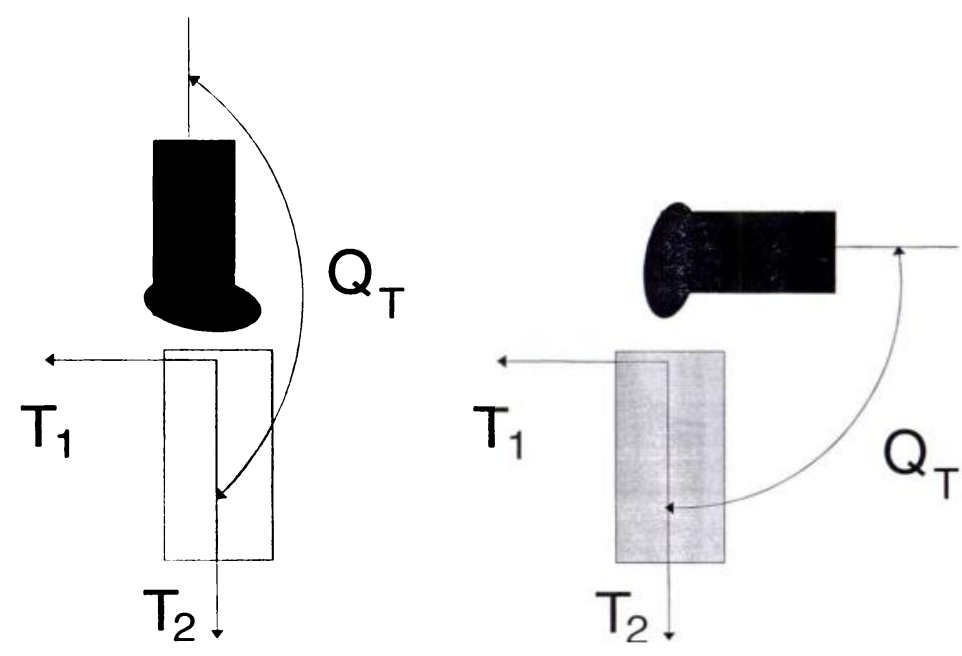

Fig. 5

Figure 3 - Patellar ligament rotation is measured with reference to a longitudinal line through the axis of the tibia at the midsagittal point. Figure 4 Patellar rotation is measured from the angle of the patellar ligament with the longitudinal axis of the patella. Figure $5-\mathrm{Range}$ of movement is measured from the axis of both the tibia and femur.

and patella, relative to the tibia, were obtained by using successive photographs superimposed in sequence.

A mapping technique was used to collect data points. For the analysis, the tibia was assumed to be fixed in the system with the femur and the patella rotating and translating relative to the midpoint in the sagittal plane of the proximal tibial surface. The patellar ligament rotation angle was measured from the line perpendicular to the proximal tibial joint surface intersecting with a line placed over the patellar ligament $\left(Q_{L}\right.$; Fig. 3). The patellar rotation angle was measured between the patellar ligament line and a line through the vertical axis of the patella $\left(Q_{p} ;\right.$ Fig. 4). Although the patella is in contact with the femur, it rotates relative to the tibia and translates relative to a fixed point on the tibia. Maximum knee flexion was calculated for each patient (diff $\mathrm{Q}_{\mathrm{T}}$; Fig. 5).

\section{RESULTS}

Initially, the fluoroscopic examinations were made on four normal knees to determine normal kinematics (Fig. 6). At full extension, the femur contacts the tibia $6 \pm 1 \mathrm{~mm}$
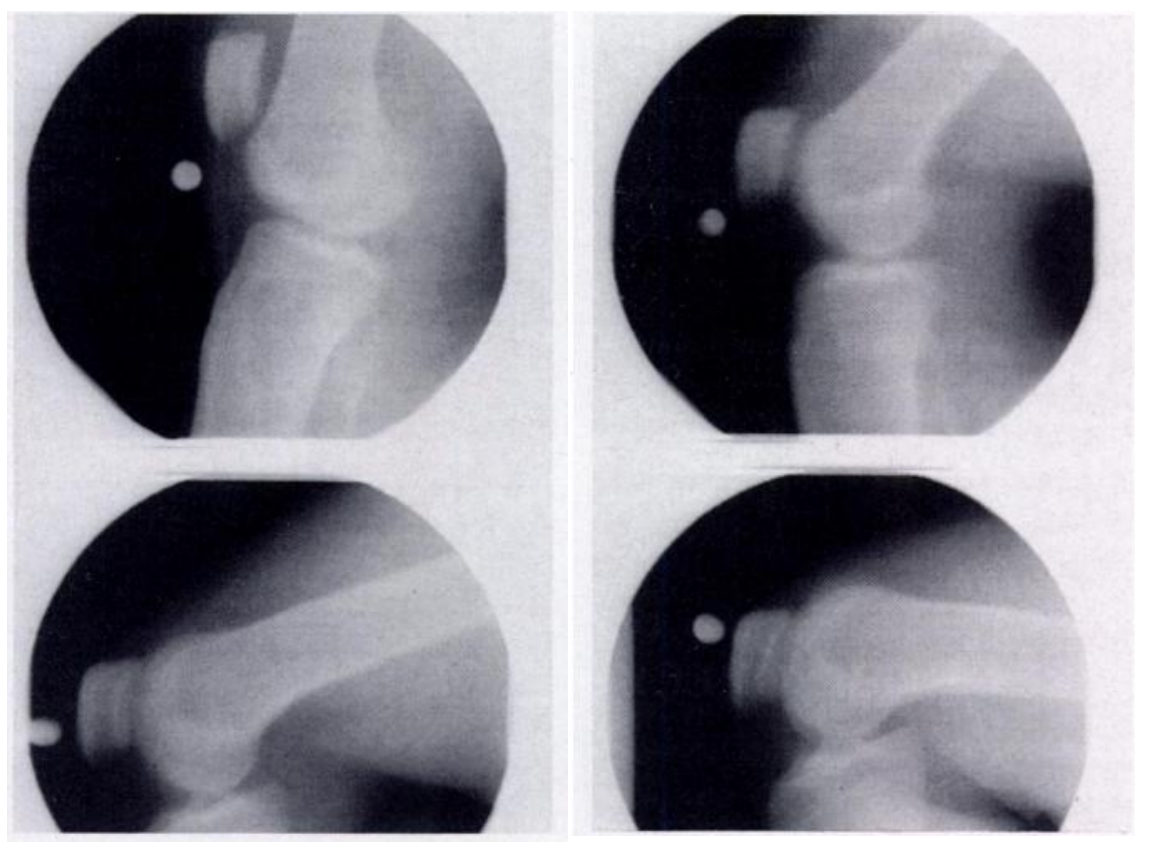

Fig. 6

Fluoroscopic views of a normal knee through the range of movement. 

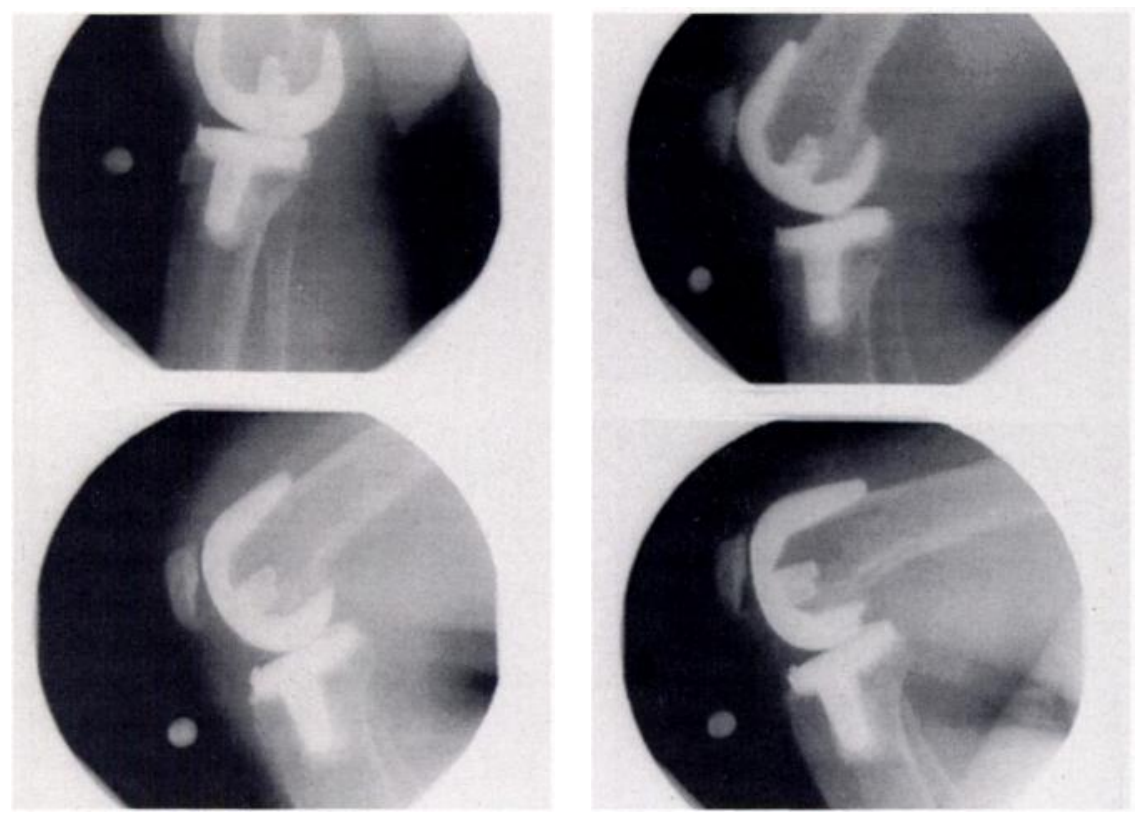

Fig. 7

Fluoroscopic views of a PCR TKA showing the tendency to translate anteriorly with increasing flexion under weight-bearing conditions.

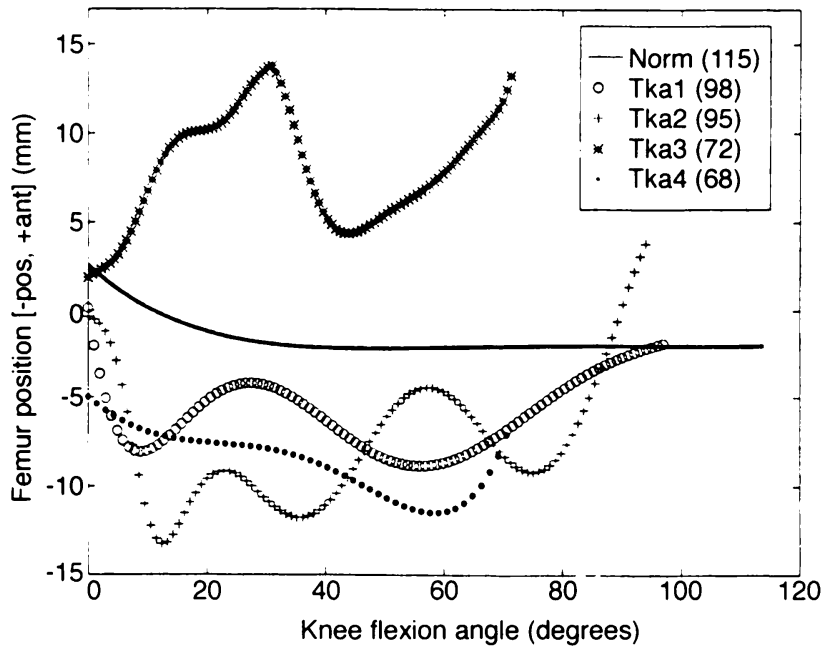

Fig. 8

The five prostheses show erratic movement and a tendency to translate forward with increasing flexion compared with the normal knee. There are various starting points in extension.

anterior to the midline in the sagittal plane of the tibial joint surface, but translates to $2 \pm 1 \mathrm{~mm}$ posterior during flexion. During most of the flexion cycle, this contact point remains relatively near the midline. Fluoroscopic photographs of PCR knees showed that during full extension, femorotibial contact points start $10 \pm 5 \mathrm{~mm}$ posterior to the midsagittal line. Unlike the normal knee which rotates posteriorly during flexion, all PCR TKAs translate to a point $5 \pm 3 \mathrm{~mm}$ anterior to the midsagittal point (Fig. 7). In contrast with the normal knees which had a smooth motion during the flexion cycle, the implanted knees had a 'jerky' discontinuous motion (Fig. 8).
Analysis of patellar ligament rotation and patellar rotation showed that the normal knees had a constrained motion so that there was only one possible patellar ligament angle and patellar angle with respect to each knee flexion angle. Patellar tracking was smooth and continuous when seen in the lateral view. Patellar function after patellar resurfacing in PCR TKA showed abnormal tracking with a pie-shaped gap opening at the distal pole of the patella as the patellar prosthesis articulated on the more superior surface of the dome. In certain cases a gap existed between the patella and the anterior femoral flange in full extension. During the dynamic video, the patella would slam into the anterior femur at the beginning of knee flexion. In some cases, the patellofemoral contact point was considerably lower than the contact position in normal knees at a similar flexion angle.

The patellar ligament rotation angle in normal knees started at $16^{\circ}$ in extension and progressed to $0^{\circ}$ in flexion. Prosthetic knees had a decreased angle in extension which also progressed to $0^{\circ}$ with flexion (Fig. 9). Patellar axis rotation increased in prosthetic knees in a similar fashion to normal knees, but was greater than normal in full flexion (Fig. 10).

Normal knee flexion averaged $118^{\circ}$ (110 to 137). For PCR TKA, the maximum weight-bearing knee flexion achieved was $98^{\circ}$. In all five groups there was at least one patient who could not flex more than $70^{\circ}$ due to discomfort. The mean prosthetic knee flexion was $90 \pm 5^{\circ}$ when these abnormal cases were excluded.

\section{DISCUSSION}

In the normal fully extended knee, the femoral contact position is anterior to the midline of the tibia. The patella 


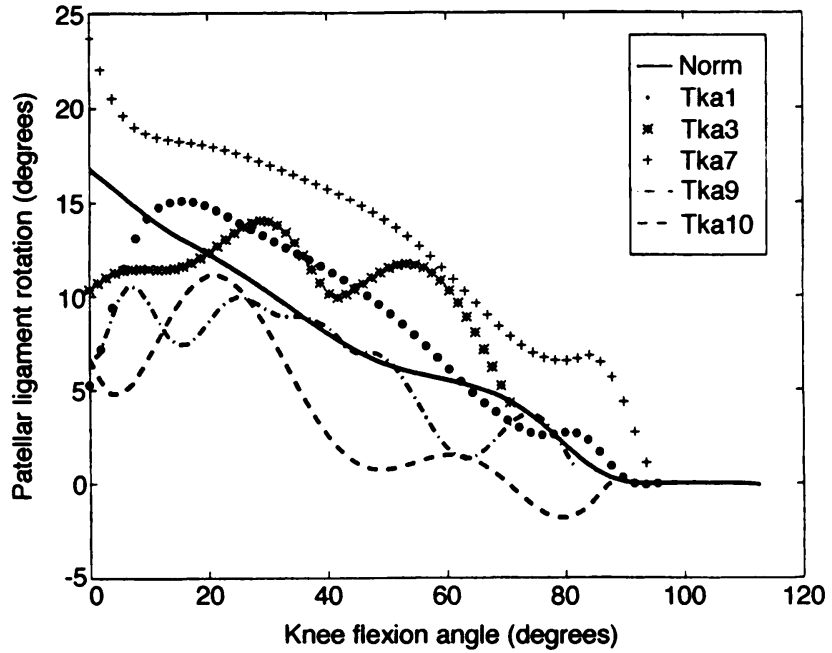

Fig. 9

In the TKAs the patellar ligament shows very erratic curves usually starting at angles greater than normal but moving to $0^{\circ}$ at full flexion.

comes into firm contact with the femur as the knee flexes; this contact remains throughout flexion.

Our study showed that PCR TKAs differed from normal knees with respect to femorotibial contact position, patellar rotation, and range of motion. Loss of the anterior cruciate ligament may be an important factor which may explain these abnormalities. Other authors have also found that knee kinematics change dramatically when the ACL is sacrificed in TKA (Goodfellow and O'Connor 1986; Weaver, Derkash and Greenwald 1993).

In the normal knee which is pulled anteriorly in full extension, the ACL is maximally loaded in extension while the PCL is minimally loaded. The PCL in TKA, without the restraint of the ACL, tends to pull the femur posteriorly during full extension, hence the posterior femorotibial contact position. On weight-bearing, the normal posteriorly directed shear force on the tibial component causes the prosthetic femur to translate anteriorly; in the normal knee, a roll-back phenomenon would be seen. Our observations showed this abnormal translation to be a 'jerky' cogwheel motion and a comparison with subsequent trials showed that virtually no curve was reproducible in any prosthetic knee. We did not find any PCR knee prosthesis which differed from this typical kinematic profile and we believe that these findings are not related to a specific design.

Fluoroscopy has demonstrated that implanted patellae are abnormally mobile compared with the constrained motion seen with the normal patella. In the normal knee, the contact position of the femur begins anterior to the midline of the tibia and the patella is in firm contact with the femur at the beginning of the flexion cycle. As the knee flexes, this firm contact remains throughout the movement. In the PCR TKA, the femur is pulled posteriorly during full extension such that the patella is not in firm contact at the

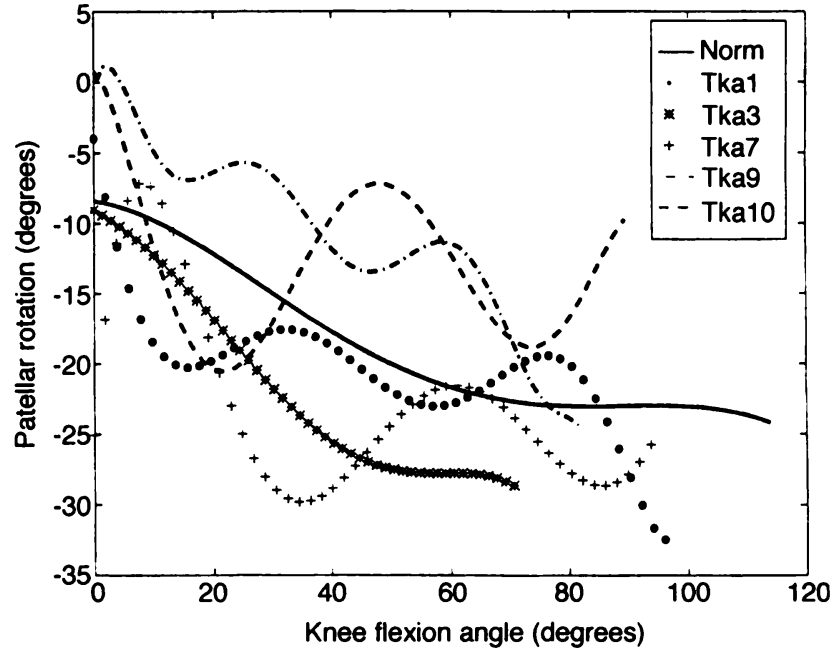

Fig. 10

In the TKAs patellar rotation shows erratic curves and a tendency to finish at a greater value than normal.

start of early flexion. In certain cases, there was a definite gap between the posterior surface of the implant and the anterior femoral flange. The prosthetic resurfaced patella did not follow a consistent path with movement. With increasing flexion, a distal gap was noted as the contact surface tended to shift to an abnormally superior position.

The range of movement in TKA was significantly less than in normal knees, an observation which may be related in part to the loss of normal roll-back. Other studies have reported a higher TKA range of movement than in our study, but it is presumed that in these the patients were examined non-weight-bearing (Wright et al 1990; Maloney and Schurman 1992; Callahan et al 1994), and the investigator can manipulate the tibia, finding the path of least resistance. In our study, the patients were fully weightbearing and knee movements followed the path prescribed by articular and soft-tissue constraints.

Lateral liftoff in midflexion was typical in many of these knees and reflects an abnormality of lateral joint laxity. We were unable to quantitate this phenomenon exactly by our technique, but were quite convinced of its presence. The implication is that flat condylar components in the coronal plane will edge-load in the medial compartment. Convex surfaces typical of earlier condylar designs would be more favourable in this regard.

The accuracy of this fluoroscopic technique for gathering positional data is verified by the fact that the curves generated in the normal patients were virtually superimposable. Slight rotation of the limb, or changes in tube angle of the X-ray beam, did not therefore have a measurable effect. We did not verify the reproducibility of the femorotibial contact measurements although determination of the point of contact of the tibial polyethylene insert appeared to be obvious in most instances. 
Our method provides a more realistic understanding of joint kinematics compared with anatomical models or theoretical predictions. Banks and Hodge (1993), with very few examples, performed a similar review analysing a meniscal-bearing implant. Their results and those of a radiological study by Kim (1994) are consistent with our findings.

Our study raises several important questions regarding existing PCR TKA. Physiological roll-back has not been demonstrated and its absence is likely to reflect anteriorcruciate deficiency, alteration of the normal joint line, or other subtle changes which modify kinematics. We are concerned by the magnitude of abnormal translation which can only magnify the degree of shear forces at the articulation. Range of movement, measured under load, may be a better functional assessment. Finally, the patellar constraint found in normal knees does not appear to be reproduced by existing patellar resurfacing designs.

One or more of the authors have received or will receive benefits for personal or professional use from a commercial party related directly or indirectly to the subject of this article.

\section{REFERENCES}

Andriacchi TP, Stanwyck TS, Galante JO. Knee biomechanics and total knee replacement. J Arthroplasty 1986;1:211-9.

Banks SC, Hodge WA. Direct measurement of 3D knee prosthesis kinematics using single plane fluoroscopy. Proceedings of the Orthopaedic Research Society 1993;18:428.

Bartel DL, Bicknell VL, Wright TM. The effect of conformity, thickness and material on stresses in ultra-high molecular weight components for total joint replacement. J Bone Joint Surg [Am] 1986;68-A:1041-51.

Blunn GW, Walker PS, Joshi A, Hardinge K. The dominance of cyclic sliding in producing wear in total knee replacements. Clin Orthop 1991;273:253-60.

Brown TD, Shaw DT. In vitro contact stress distribution on the femoral condyles. J Orthop Res 1984;2:190-9.

Callahan CM, Drake BG, Heck DA, Dittus RS. Patient outcomes following tricompartmental total knee replacement: a meta-analysis. JAMA 1994;271:1349-57.

Collier JP, Mayor MB, Surprenant VA, et al. The biomechanical problems of polyethylene as a bearing surface. Clin Orthop 1990;261:107-13.

Collier JP, Mayor MB, McNamara JL, Surprenant VA, Jensen RE. Analysis of the failure of 122 polyethylene inserts from uncemented tibial knee components. Clin Orthop 1991;273:232-42.

EI Nahass B, Madson MM, Walker PS. Motion of the knee after condylar resurfacing. Proceedings of the Orthopaedic Research Society 1990;15:476.

Goldberg VM. Controversies of total knee arthroplasty: Bristol-Meyers/ Zimmer Orthopaedic Research Symposium Series. New York, Raven Press, 1991:61-73.
Goodfellow JW, O'Connor J. Clinical results of the Oxford knee: surface arthroplasty of the tibiofemoral joint with a meniscal bearing prosthesis. Clin Orthop 1986;205:21-42.

Grood ES, Shastri R, Hopson CN. Analysis of retrieved implants: crystallinity changes in ultrahigh molecular weight polyethylene. Journal of Biomedical Materials Research 1982;16:399-405.

Hsu RWW, Himeno S, Coventry MB, Chao EYS. Normal axial alignment of the lower extremity and load-bearing distribution at the knee. Clin Orthop 1990;255:215-27.

Insall JN, Lachiewicz PF, Burstein AH. The posterior stabilized condylar prosthesis: a modification of the total condylar design: two to four-year clinical experience. $J$ Bone Joint Surg [Am] 1982;64-A:1317-23.

Kilgus DJ, Moreland JR, Finerman GA, Funahashi TT, Tipton JS. Catastrophic wear of tibial polyethylene inserts. Clin Orthop 1991:273:223-31.

Landy MM, Walker PS. Wear of ultra-high-molecular-weight polyethylene components of 90 retrieved knee prostheses. J Arthroplasty 1988;3 [Suppl]:S75-85.

Mahoney OM, Noble PC, Alexander JW, Rhoads DD, Tullos HS. Posterior cruciate function following total knee replacement: a biomechanical study. Proceedings of Orthopaedic Research Society 1990;15:478.

Maloney WJ, Schurman DJ. The effects of implant design on range of motion after total knee arthroplasty. Clin Orthop 1992;278:147-52.

Müller W. The knee: form, function and ligament reconstruction. New York, etc: Springer-Verlag, 1983.

Nittsu M, Akisada M, Miyakawa S. Moving knee joint: technique for kinematic MR imaging. J Radiol 1990;174:569-70.

Nisell R, Nemeth G, Ohlsen H. Joint forces in extension of the knee: analysis of a mechanical model. Acta Orthop Scand 1986;57:41-6.

O'Connor J, Shercliff T, FitzPatrick D, Biden E, Goodfellow J. Mechanics of the knee. In: Daniel DM, Akeson WH, O'Connor JJ, eds. Knee ligaments: structure, function, injury and repair. New York: Raven Press, 1990:201-37.

Rose RM, Cimino WR. Exploratory investigations on the structure dependence of the wear resistance of polyethylene. Wear 1982;77:89-104.

Schlepckow P. Three-dimensional kinematics of total knee replacement systems. Arch Orthop Trauma Surg 1992;111:204-9.

Stein ID, Granik G. The human tibia: a simplified method of radiographic analysis of its cross-section, with anthropometric correlations. Ann Biomed Eng 1979;7:103-16.

Stern SH, Insall JN. Posterior stabilized prosthesis: results after follow-up of nine to twelve years. J Bone Joint Surg [Am] 1992;74-A:980-6.

Swany MR, Scott RD. Posterior polyethylene wear in posterior cruciate ligament-retaining total knee arthroplasty: a case study. J Arthroplasty 1993;8:439-46.

Weaver JK, Derkash RS, Greenwald AS. Difficulties with bearing dislocation and breakage using a movable bearing total knee replacement system. Clin Orthop 1993;290:244-52.

Wongchaisuwat C, Hemami H, Buchner HJ. Control of sliding and rolling at natural joints. J Biomech Eng 1984;106:368-75.

Wright J, Ewald FC, Walker PS, et al. Total knee arthroplasty with the kinematic prosthesis: results after five to nine years: a follow-up note. J Bone Joint Surg [Am] 1990;72-A:1003-9.

Yamaguchi GT, Zajac FE. A planar model of the knee joint to characterise the knee extensor mechanism. J Biomech 1989;22:1-10. 